\title{
ARTE CONTEMPORÁNEO Y STEAM EN LA FORMACIÓN DE MAESTROS DE EDUCACIÓN PRIMARIA: INTERSECCIONES ARTE Y CIENCIA
}

\author{
Francisco Javier Serón Torrecilla \\ Universidad de Zaragoza. Dpto Didáctica de las Ciencias Experimentales \\ Escuela Superior de Diseño de Aragón. Dpto. Fundamentos Científicos de Diseño \\ Víctor Murillo Ligorred \\ Universidad de Zaragoza. Dpto. Expresión Musical, Plástica y Corporal
}

\section{Resumen}

El enfoque STEM ha devenido en la última década en un nuevo acrónimo STEAM, en el cual, Arte y Diseño se presentan con todo su potencial. Elementos como el pensamiento creativo y crítico, asociados a dichas dimensiones, parecen ser el soporte complementario para afrontar la enorme complejidad del siglo XXI, entre las que se encuentra la enseñanza y, en el caso concreto presentado, la formación de maestros y maestras en educación primaria. En este artículo se aborda a través de una experiencia que se ha desarrollado durante los últimos cuatro cursos académicos, el impacto del trabajo sobre la base de los discursos que nacen de las expresiones del arte contemporáneo en un contexto didáctico en el que intervienen de forma interdisciplinar distintas áreas. A partir de la selección de varios casos, y el análisis de los productos de arte y ciencia resultantes, se analiza cómo el arte contemporáneo en su imbricación con los STEAM, puede facilitar la comprensión conceptual de fenómenos como son la luz y el color que implican a dos áreas de la didáctica.

\section{Palabras clave: STEAM; ARTE CONTEMPORÁNEO; CIENCIA; INTERDIS- CIPLINARIEDAD; DIDÁCTICA}

\section{CONTEMPORARY ART AND STEAM IN THE TRAINING OF PRIMARY EDUCATION TEACHERS: ART AND SCIENCE INTERSECTIONS}

\section{Abstract}

The STEM approach has evolved over the last decade into a new acronym STEAM, in which Art and Design are presented in their full capacity. Elements such as creative and critical thinking, associated to these dimensions, seem to be the complementary support to face the enormous complexity of the 21st century, among which we find teaching and, in the specific case presented, pre-service teachers in primary education. Through an experience that has been developed over the last four academic years, this article addresses the impact of work based on the discourses that arise from contemporary art expressions in a didactic context in which different areas intervene in an interdisciplinary manner. Based on the selection of several cases, and the analysis of the resulting art and science products, we analyze how contemporary art, in its imbrication with the STEAMs, can facilitate the conceptual understanding of phenomena such as light and color that involve two areas of didactics.

Keywords: STEAM; CONTEPORARY ART; SCIENCE; INTERDISCIPLINARITY; DIDACTIC

\footnotetext{
Serón Torrecilla, Francisco Javier; Murillo Ligorred, Víctor. 2020. "Arte contemporáneo y STEAM en la formación de maestros de educación primaria: Intersecciones arte y ciencia“. AusArt 8 (1): 65-76. D0I: 10.1387 /ausart.21462
} 


\section{INTRODUCCIÓN}

La menor presencia curricular del arte, no ha supuesto que se siga reivindicando su capacidad en la actividación de elementos cognitivos, algo fundamental en los procesos de enseñanza y aprendizaje desde una perspectiva más holística. En este sentido, la incorporación del Arte al paradigma de la estrategias STEM (Science, Technology, Engineering and Mathematics), dado su aparente fracaso, va más allá de redimensionar el acrónimo, ahora STEAM, puesto que introduce elementos esenciales en cuanto a lo que significan para los procesos de aprendizaje, al presentarse vehiculados desde la dimensión artística.

El análisis de experiencias previas en la formación científica en el Grado de Maestro en Educación Primaria de recursos didácticos del Área de Didáctica de la Expresión Visual y Plástica (en adelante EVyP) y viceversa, han ayudado a valorar las carencias y posibilidades de enseñanza de nuestros estudiantes a partir por ejemplo de aprendizajes basados en proyectos. Desde este análisis reflexivo del propio ejercicio docente, nace la propuesta que se presenta en este artículo con un objetivo claro, potenciar las relaciones para la mejora de aprendizajes más globalizados. En este caso, y por la complejidad de los contenidos trabajados, como son la naturaleza de la luz y el color, de gran impacto en la construcción de la realidad, se ha tratado de articular en torno a expresiones del arte y la cultura contemporánea un discurso que les ayude a experienciar nuevos enfoques que, por otro lado, ya se están implementado en las aulas de primaria y que, por tanto, nos sitúa en el deber como formadores de los futuros egresados en magisterio, prepararlos para su función en el ejercicio profesional.

Los resultados se presentan a partir del estudio de casos. Se ha empleado un análisis descriptivo del discurso proveniente de distintas herramientas de valoración del proyecto, cuaderno de campo, memorias del proyecto, presentaciones de los estudiantes, etc.

En primer lugar se sustenta la propuesta a partir de un breve análisis del problema en relación al uso STEAM y la incorporación del arte contemporáneo como elemento de enseñanza y aprendizaje en el fomento de las relaciones interdisciplinares. A continuación se apunta los objetivos, abordando los casos seleccionados de un modo descriptivo. Para finalizar se analizan algunos de los resultados a partir de una reflexión sobre el impacto positivo y la coherencia con lo señalado por diversos autores en el marco teórico. Se concluye 
estableciendo la necesidad de seguir promoviendo dichos enfoques por su complementariedad en la formación y el trabajo por proyectos y en relación al futuro ejercicio docente de nuestros estudiantes.

\section{FUNDAMENTACIÓN TEÓRICA}

Arnheim (1993) afirma que el arte, a pesar de la cada vez menor presencia en los currículos educativos, presenta un enorme potencial formativo para todas las áreas desde los procesos cognitivos que activa, facilitadores en la construcción de conceptos.

El autor aprecia un necesario ejercicio de vincular las materias del intelecto con las de la intuición. En ese mismo sentido, otros autores como Gardner $(1987 ; 1990)$ han asemejado el pensamiento complejo del arte al de la ciencia o las matemáticas. Por su carácter simbólico y la necesidad del ser humano de operar con símbolos, fomentarían la construcción de mundos con significados mucho más integradores.

Otro autor fundamental en la reivindicación del papel del arte es Goodman (1984) quien ha situado el arte junto con la física y la filosofía en su capacidad de construir las distintas versiones del mundo que facilitan su comprensión, desde un equilibrio entre todas las dimensiones del conocimiento y sin predominio epistemológico entre ellas.

En cuanto a la capacidad de activar elementos cognitivos, otros autores (Eisner 1994; Efland 2004) han señalado la necesidad de superar entornos limitantes en los que ha predominado hasta ahora la palabra y los números, afirmando la urgente necesidad de reconocer a la imaginación como una herramienta positiva de cognición para la educación en general, reforzando su potencialidad como elemento interdisciplinar.

En el caso de la incorporación de arte contemporáneo como recurso, en ámbitos de la práctica museística y artística se ha reconocido el gran desconocimiento que existe para el alumnado y el profesorado (Berrocal 2005). En este último caso, se aprecia extrañeza, miedo, incomprensión y, en la mayoría de los casos, inseguridad al tratar con dichos recursos. 
Desde la experiencia de muchos años como directora del proyecto Expressart museo portátil para el Museo de Arte Contemporáneo de Barcelona en educación infantil y primaria con resultados muy positivos, Marta Berrocal ha señalado en relación a los objetivos lo siguiente: "el objetivo del material es proporcionar recursos para trabajar en el aula cuestiones y temáticas diversas desde un enfoque competencial que parte del arte como fuente generadora de aprendizaje (2018).

Igualmente, autores como (Tárrega 2014; Ching 2015) han apuntado hacia la urgente necesidad de alfabetizar a nuestros maestros y estudiantes en los códigos y lenguajes del arte contemporáneo. Un requerimiento que nace de la necesidad de entender una sociedad, cada vez, más compleja y en la que el arte contemporáneo ofrecería recursos para entender conceptos que se insertan en ella desde la cultura tecno-científica.

De este modo, con los elementos presentes sobre el valor cognitivo del arte, así como el arte contemporáneo en particular para la construcción de una mejor comprensión de la dimensión humana y la sociedad actual, sería lógico pensar la necesidad de encontrar enfoques capaces de movilizar todas esas variables. Así, el enfoque STEAM, que nace de esa misma reflexión ante el aparente fracaso de los STEM en su objetivo de promover las vocaciones tecno-científicas, presentaría dichas características.

Diversos autores (Maeda 2013; Yakman 2008; Yakman \& Lee 2012; Taylor 2016; Taylor \& Taylor 2017) provenientes de distintos ámbito ya habrían señalado, en consonancia con lo anteriormente expuesto, la capacidad del arte para proporcionar herramientas para entender nuestra compleja realidad y la construcción de significados.

El arte dejaría de ser una dimensión espectadora para facilitar el tránsito del aprendizaje en una adquisición de conocimientos y construcción de conceptos de carácter transdisciplinar, en los que prima, ante todo, la cultura humana (Belardo, Burrows \& Dambekalns 2017). Pérez Tudela (2015) ha afirma en relación a la dimensión creativa en los STEAM que "el arte fomenta la innovación y el diseño, el desarrollo de la curiosidad, la imaginación y la búsqueda de soluciones diversas a un único problema" algo no solo necesario, sino imprescindible para el avance de la ciencia y de la tecnología y, en general, de la sociedad. 


\section{OBJETIVOS Y DESCRIPCIÓN DE LA PROPUESTA}

El objetivo fundamental de esta propuesta, que con los cambios necesarios se inicia durante el curso académico 2015/2016, y en la que a lo largo de los cuatro cursos siguientes ha supuesto la participación de más de mil estudiantes del Grado en Magisterio de Enseñanza Primaria en su segundo curso, era utilizar un recurso como es el arte contemporáneo en el marco formativo de la didáctica de la EVyP y de las ciencias experimentales, en contexto y tradición en el que ambas áreas tienen de aprendizaje por descubrimiento/indagación en el marco de los trabajos prácticos.

A partir de analizar puntos en común, en relación a la enseñanza y aprendizaje de contenidos conceptuales complejos, como son la luz y sus fenómenos relacionados, se diseñó una propuesta de aprendizaje basada en proyectos de enfoque STEAM que, tomando como referencia el arte contemporáneo y el impacto, análisis y reflexión de los artistas en torno a estos contenidos, permitiera trabajar con los estudiantes en la mejora de la comprensión conceptual.

Hay que destacar de forma breve que la luz como fenómeno, no se aborda siempre de forma complementaria desde ambas áreas y que produce muchas dudas entre el alumnado siendo, por otra parte, un elemento esencial en la construcción de realidades que abarcan aspectos físicos, químicos, biológicos, artísticos, estéticos, psicológicos, etc.

Señalamos como resultado del proyecto que los estudiantes elaboran a lo largo del cuatrimestre un producto artístico-científico. Dicho producto tiene como premisa el poseer un valor didáctico, contando con su capacidad de transferir su conocimiento a un aula de primaria como artefacto de enseñanza y aprendizaje de los contenidos, en este caso, de la luz y el color. Al mismo tiempo, el objetivo se sitúa en la elaboración de su producto, para que nuestro alumnado mejore su capacidad de análisis, discusión y argumentación en torno a temas fundamentales de su realidad contemporánea, competencias transversales y comunes a todas las áreas que formar parte del currículo del grado.

Para este artículo, y por la cantidad de productos obtenidos, se ha tomado la decisión de analizar tres de ellos de los cursos 2017/2018 y 2018/2019, que implican, por otra parte, a dos figuras destacadas del arte contemporáneo. La 
selección de estos dos casos se ha sustentado en el análisis del progreso de los estudiantes a lo largo de las distintas etapas de desarrollo del proyecto, así como en los resultados alcanzados en la elaboración de un objeto por su potencial didáctico para ambas áreas. El valor de las propuestas presentadas se ha establecido a partir de distintas herramientas de evaluación y por parte de distintos agentes, docentes de ambas áreas y los propios compañeros a partir de un proceso de co-evaluación que incorpora preguntas durante la defensa de su propuesta.

Algunos de los aspectos fundamentales que caracterizan al proyecto se centran en la selección previa de artistas contemporáneos, realizada por el Área de Expresión Visual y Plástica durante las dos primeras ediciones del proyecto, para facilitar el trabajo de los estudiantes. En el primer caso, la selección se realizó a partir de establecer ejes fenomenológicos en los que la luz cobra especial importancia en el desarrollo de la obra, destacando los siguientes artistas: David Hockney, Gerhard Ritchter, Man Ray, Bill Viola, Naum Gabo o Pablo Gargallo.

En el caso del curso académico 2018/2019 se introduce, además, la perspectiva de género, habida cuenta de que el $90 \%$ del estudiantado son mujeres destacando las siguientes artistas: Dara Bimbaum, Yayoy Kusama, Mona Hatoum, Cristina Ghetti, Mayte Alonso, Gema Ruperez, Fatima Mazmouz, Amal Kenawy.

\section{PRESENTACIÓN Y ANÁLISIS DE LOS CASOS SELECCIONADOS}

El proyecto presenta múltiples etapas que son guiadas por las y los docentes de ambas áreas, donde los grupos de cuatro estudiantes van analizando desde ambas dimensiones del conocimiento, los fenómenos a partir de la selección de un artista y en el proceso de construcción de su producto.

\section{DAVId HOCKNEY: LUZ Y ÓPTICA CONTEMPORÁNEA}

En este apartado se citan dos casos que tienen cómo marco de referencia las obras del artista inglés David Hockney, Retrato de un artista de 1972 y 
Nicholas Wilder Studying Picasso de 1982. En estas obras, la luz juega un papel fundamental en la construcción de atmósferas y realidades, un análisis que llevan a cabo los estudiantes en un proceso guiado por los docentes. De esta forma, el relato llega a transcender la propia dimensión del arte contemporáneo. Nos hablan de la realidad y cómo en ella se insertan otras realidades provenientes de distintos tipos de conocimientos. Fenómenos científicos que alumbran perspectivas, colores o deformaciones que les llevan, a los estudiantes a profundizar en el análisis o incluso en plantearse qué es la realidad, cuestión muy en boga en la introducción de tecnología como la realidad virtual o aumentada. Se trataría por tanto de cómo sus productos acaban siendo modelos explicativos de carácter didáctico (ver figuras 1 y 2 ).

En ambas propuestas existe una reconstrucción reflexiva a partir de la obra del artista en ese contexto didáctico que da servicio a la construcción de conceptos complejos vinculados con la luz, como son la reflexión, refracción y que configuran en cierto modo las realidades. Existe por tanto una intencionalidad y aporte propio de los estudiantes.

En la figura 1, el hecho de salir de las dos dimensiones hace que la escena cobre vida y se referencie a ese punto de luz móvil que sitúan sobre el arco del personaje principal. Un relato, que a pesar de expresar cierto mimetismo con el original, acaba constituyendo otros puntos de vista y perspectivas, como así han expresado las estudiantes.

En el producto que se muestra en la figura 2, la construcción del objeto presenta un punto inicial de indagación y exploración de los materiales y sus efectos. Más allá de la propuesta y el análisis de la obra de Hockney, los
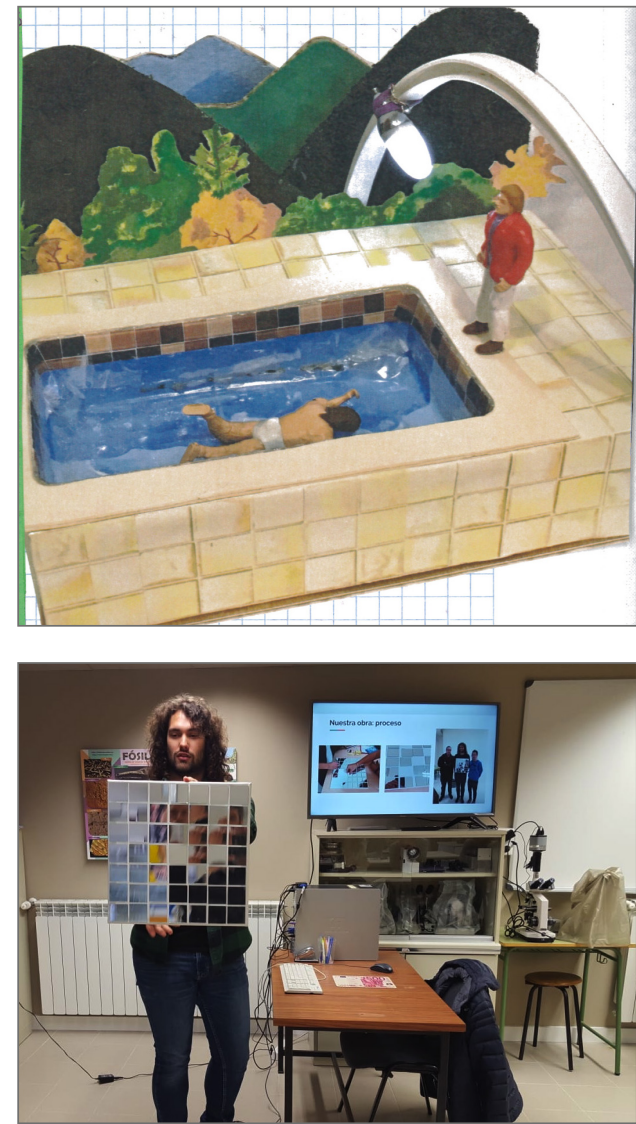

Figuras 1 y 2: Productos artístico-científicos elaborados a partir del análisis, reflexión y conceptualización por parte de los maestros y maestras de las obras de Hockney. 
estudiantes juegan con las ilusiones que genera su configuración, así como las percepciones al modificar el ángulo de visión, poniendo en jaque el significado de lo real y lo reflejado. Y es, de esta forma que, en su discurso se presentan esos objetivos iniciales de analizar y reflexionar sobre la realidad y los elementos que la afectan, entre los que se encuentra la luz, desde un punto de vista físico, además de su componente psicológico y biológico.

\section{Arte contemporáneO, Perspectiva de género y SOCiedad}

Desde esta mirada, las estudiantes sustentas la elaboración de su producto en el análisis de la biografía y la obra de la artista egipcia Amal Kenawy, fallecida en 2012. En la figura 7 una instantánea de su instalación performática. Una obra que resume su contexto filosófico y estético.

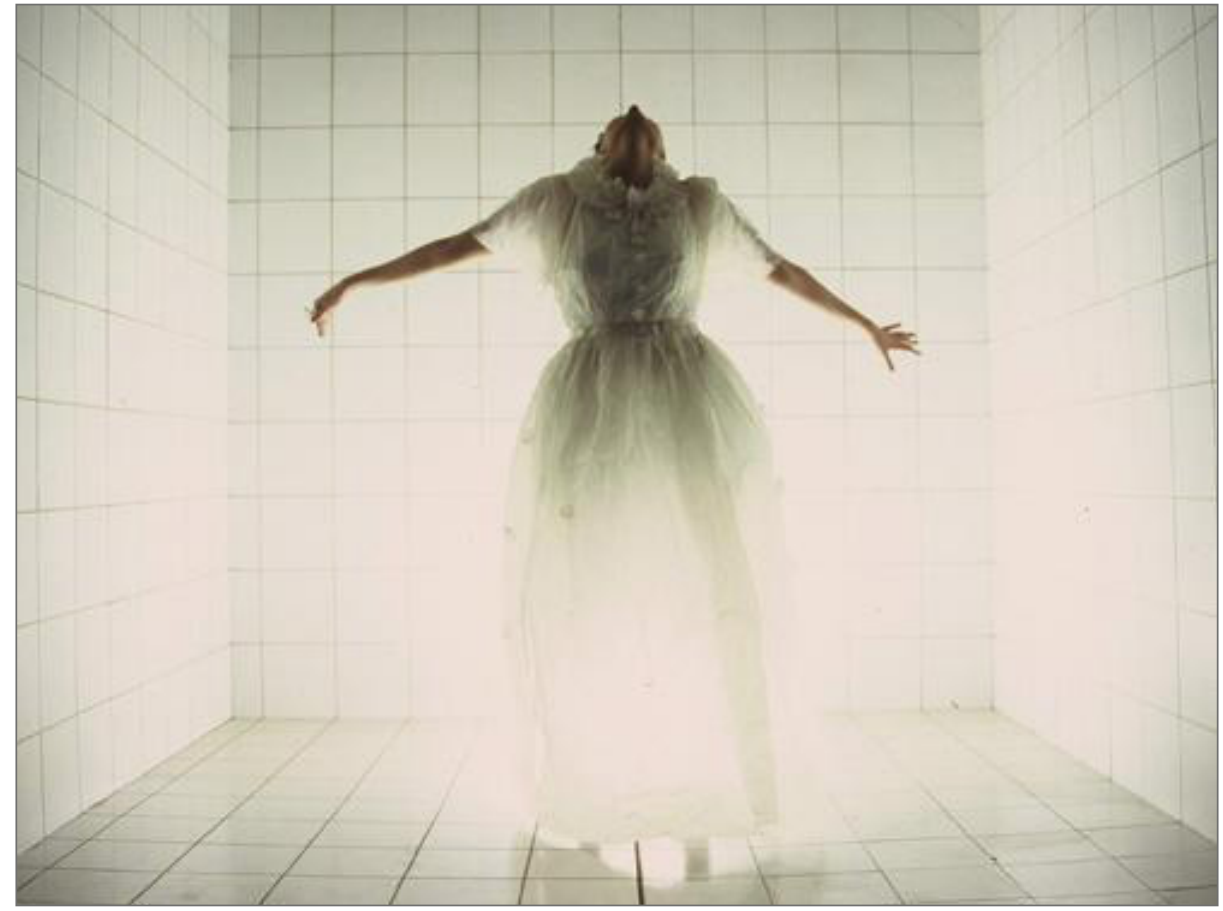

Figura 7: Amal Kenawy: The journey (2004)

A partir de la obra titulada The journey (2004), las estudiantes analizan y reflexionan en su propuesta partiendo de las afecciones que introduce la presencia de la luz como un elemento transformador de la realidad. En concreto, 
señalan como el discurso de la artista se ve influenciado por este marco conceptual. Al mismo tiempo, las estudiantes profundizan en su biografía, resaltando su compromiso social, feminista y transcultural.

Para entender el análisis de nuestras estudiantes y su producto, tan solo apuntar unas palabras seleccionadas:

No concibo mi arte como el de una feminista en el sentido tradicional de la palabra, sino como el de una artista.... Me importa la memoria y emociones humanas como el deseo y la violencia, entre otros sentimientos. Estoy intentando encontrar un lenguaje capaz de llegar al receptor, un lenguaje que no esté sujeto a una determinada cultura, oriental u occidental.

(Kenawy 2007- Sirian Women

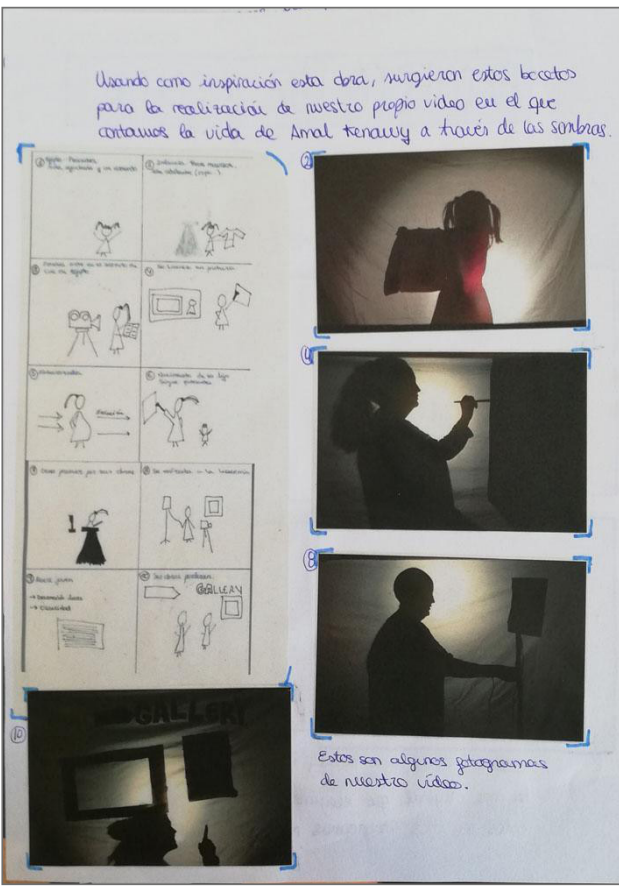

Figura 8: Imagen proveniente del cuaderno del proyecto de las estudiantes: storyboard e imágenes de su video-performance.

Observatory)

Como señalaron en su presentación, el producto representa su propio viaje personal a partir de reconceptualizar elementos provenientes del análisis de la biografía y obra de la artista. En dicho producto performático se integran aspectos artísticos-científicos y los aspectos didácticos para la enseñanza/ aprendizaje de los niños.

\section{RESULTADOS, REFLEXIÓN Y CONCLUSIONES}

De cara al análisis, y como se ha apuntado en párrafos anteriores, se emplearon distintos instrumentos de recogida de información: memoria individual del proyecto, análisis de las presentaciones ante sus compañeros y un cuaderno 
de campo. En esta última herramienta se recogieron por parte de los docentes de ambas áreas todos los aspectos relevantes acerca de la evolución en los aprendizajes (conceptuales, cognitivos) de nuestros estudiantes y su integración en el desarrollo del producto. Una información que ante todo se tomó de las tutorías y de los talleres prácticos en los que indagaban acerca de los fenómenos plásticos y científicos.

- En relación a la memoria individual, se destaca como los estudiantes señalan los aspectos positivos en relación al uso del enfoque STEAM por un lado, así como su adecuación a un aprendizaje por proyectos. Pero, ante todo, y de forma general al margen de los casos seleccionados, apuntan a la oportunidad de trabajar de esta forma, por primera vez, y la necesidad de que su formación se adecúe a este tipo de enfoques y estrategias, que consideran más coherentes con lo que va a ser su ejercicio profesional.

- En lo que se refiere al análisis de las presentaciones, existen dos elementos que se utilizaron. Por un lado, las preguntas realizadas por sus compañeros al final de la presentación en torno a elementos de carácter didáctico y conceptual y, por otro, el grado de comprensión que se permite apreciar a lo largo de la propia presentación. Parece apreciarse una mayor seguridad al exponer conceptos vinculados con el fenómeno físico y en relación a la obra de arte y su producto. Pero es, sobre todo, este último aspecto, como su discurso, y en comparación con otras propuestas o trabajos desarrollados por ambas áreas durante el cuatrimestre, donde se ve sostenido un elevado grado de reflexión analítica, discusión reflexiva y argumentación. Elementos fundamentales en la formación y que en la mayoría de los casos tienen como punto de partida la obra de arte seleccionada por los estudiantes.

Por tanto, parece confirmarse esa pretendida mejora de, no solo aprendizajes de tipo conceptual sino de aquellos elementos que movilizan aspectos mucho más profundos de tipo cognitivo y que, por otro lado, están en concordancia con lo señalado por los autores referenciados (Eisner 1994; Efland 2004; Belardo, Burrows \& Dambekalns 2017) en cuanto a la intervención del arte, y el arte contemporáneo en particular (Tárrega \& Palau 2014), y su interacción con otras disciplinas del conocimiento, dentro del marco de la educación general. Algo similar sucede en lo que respecta al enfoque y que, como han señalado los diversos autores (Yakman 2008; Yakman \& Lee 2012; Maeda 2013; Pérez Tudela 2014; Taylor \& Taylor 2017), parecen facilitar la integración de conoci- 
miento, como señalan los estudiantes, y las metodologías activas como son los aprendizajes por proyectos.

Para concluir, señalamos que el proyecto se sitúa como una aproximación que, en su utilización del arte contemporáneo como recurso, y desde el aprendizaje basado en proyectos, parecen tener un impacto positivo en el alumnado y el equipo docente. Se debería, por tanto, insistir, y como bien han apuntado nuestros estudiantes, en incoporar este tipo de propuestas en el ámbito de la enseñanza superior universitaria.

\section{Referencias ibliográficas}

Arnheim, Rudolf. 1993. Consideraciones sobre la educación artística. Traducción de Fernando Inglés Bonilla. Barcelona: Paidós

Belardo, Christy, Andrea C., Burrows \& Lydia Dambekalns. 2017. "Partnering science and art: Preservice teachers' experiences for use in pre-collegiate classrooms". Problems of education in the $21^{\text {st }}$ Century 75(3): 215-34

Ching, Chor Leng Twardzik. 2015. "Teaching contemporary art in primary schools". Athens Journal of Humanities \& Arts 2(2): 95-110. https://www.athensjournals.gr/humanities/20152-2-3-Ching.pdf

Efland, Arthur D. 2004. Arte y cognición: La integración de las artes visuales en el currículum. Trad., Angels Mata Masó. Barcelona: Octaedro

Eisner, Elliot W. (1980) 1994. Cognición y currículum. Una visión nueva. Trad., Edith Litwin. Buenos Aires: Amorrortu. Orig.: Dewey Lecture 1980

— . 1998. El ojo ilustrado, indagación cualitativa y mejora de la práctica educativa. Traducción de David Cifuentes \& Laura López. Barcelona: Paidós

Gardner, Howard. 1987. Arte, mente y cerebro. Traducción de Gloria G.M. de Vitale. Barcelona: Paidós

— . . (1990) 1994. Educación artística y desarrollo humano. Traducción de Ferrán Meler Ortí. Barcelona: Paidós

Goodman, Nelson. (1984) 1995. De la mente y otras materias. Traducción de Rafael Guardiola. Madrid: Visor

Maeda, John. 2013. "STEM + Art = STEAM". The STEAM Journal 1(1), art. 34. https://doi. org/10.5642/steam.201301.34

Pérez Tudela, Julio D. 2015. "STEM, STEAM... ¿ ¿pero eso qué es?". Odite.ciberespiral.org, 21 abril. http://odite.ciberespiral.org/ca/comunitat/ODITE/recurs/stem-steam-pero-eso-quees/58713dbd-414c-40eb-9643-5dee56f191d3

Taylor, Peter. 2016. "Why is a STEAM curriculum perspective crucial to the 21st Century?". Invited paper presented at the Australian Council for Education Research-ACER Research Conference 'Improving STEM learning - What will it take?' 7-9 August, Brisbane 
— \& Peter C. Taylor. 2017. "Breaking down enlghtenment silos: From STEM to ST2EAM education and beyond". En Thirteen questions: Reframing education's conversation; Science, Joe L. Kincheloe \& Shirley R. Steinberg, eds. Bern: Peter Lang

Tárrega Astigarraga, Oihana \& Paloma Palau Pellicer. 2014. "La educación plástica y visual en espacios de arte contemporáneo". Fòrum de Recerca 19: 239-58

Yakman, Georgette. 2008. "STEAM Education: An overview of creating a model of integrative education". Tesis Virginia Polytechnic and State Univ.

Yakman, Georgette \& Hyonyong Lee. 2012. "Exploring the exemplary STEAM education in the US as a practical educational framework for Korea". Journal of the Korean Association for Research in Science Education 32(6): 1072-86. https://doi.org/10.14697/ jkase.2012.32.6.1072

(Artículo recibido: 08-02-20; aceptado: 19-05-20) 